\title{
No-pumping theorem for many particle stochastic pumps
}

\author{
Shahaf Asban ${ }^{1}$ and Saar Rahav ${ }^{2}$ \\ ${ }^{1}$ Faculty of Physics, Technion - Israel Institute of Technology, Haifa 32000, Israel \\ ${ }^{2}$ Schulich Faculty of Chemistry, Technion - Israel Institute of Technology, Haifa 32000, Israel
}

(Dated: October 10, 2018)

\begin{abstract}
Stochastic pumps are models of artificial molecular machines which are driven by periodic time variation of parameters, such as site and barrier energies. The no-pumping theorem states that no directed motion is generated by variation of only site or barrier energies [S. Rahav, J. Horowitz, and C. Jarzynski, Phys. Rev. Lett., 101 , 140602 (2008)]. We study stochastic pumps of several interacting particles and demonstrate that the net current of particles satisfies an additional nopumping theorem.
\end{abstract}

PACS numbers: 05.60.-k, 03.65.Vf,05.10.Gg ,82.20.-w

Molecular motors and machines are an essential component in living organisms. They perform tasks such as carrying loads, contracting muscles and many other crucial functions 1]. Many research groups are actively trying to venture beyond the motors found in nature, aiming to design and synthesize artificial molecular machines [2 6].

Since artificial machines can be designed, it is possible to operate them using new driving mechanisms which are not found in biological motors and machines. One such driving mechanism is the rectification of periodic time variation of external parameters. Due to the similarity with everyday pumps such systems are often referred to as stochastic pumps. Stochastic pumps are therefore closely related to thermal ratchets 7], but the term is more commonly used for systems with a discrete set of coarse-grained states. The dynamics of stochastic pumps have been investigated extensively in recent years [8 13], see also the reviews by Sinitsyn 14 and Astumian 15] for an overview.

Motivated by a beautiful experiment on catananes 16], and by work focused on an adiabatically driven model [17], a no-pumping theorem (NPT) for stochastic pumps was found [18]. This non adiabatic result identifies driving mechanisms which will not lead to directed motion. In a parallel development, Chernyak and Sinitsyn [19] showed how to generalize the NPT to account for the topology of the network of transitions between states. Due to its simple structure, and somewhat non intuitive result, the NPT has generated considerable interest $20-$ 24]. All this body of work was focused on the stochastic dynamics of a single particle. The aim of this letter is to investigate the validity of the NPT for many particle stochastic pumps, where several interacting particles jump between a set of binding sites. We demonstrate that an NPT holds for this many particle system for any local interaction.

Single particle NPT- Consider a system which makes sudden transitions between a set of coarse-grained states, labeled by $\alpha, \beta, \gamma, \cdots$ The transition are assumed to be Markovian and are characterized by transition rates

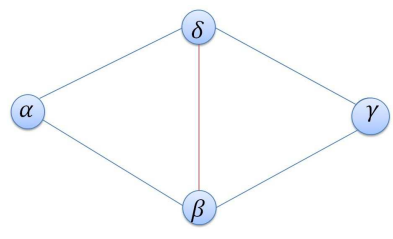

(a)

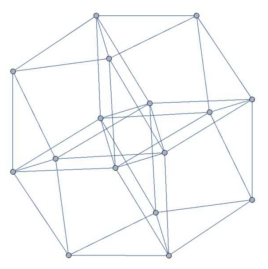

(c)

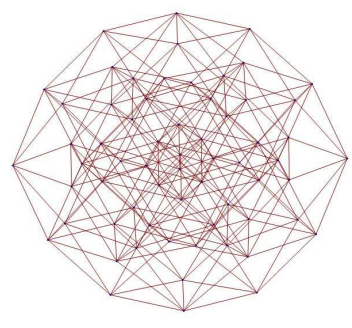

FIG. 1: The graph representation of stochastic pumps with 4 sites and 5 bidirectional transitions: (a) the single particle pump, (b) and (c) the product graph representing a system with 2 and 3 particles respectively. The nodes of the product graphs correspond to many particle states such as $(\alpha, \gamma, \alpha)$.

$R_{\beta \alpha} \geq 0$ (for $\alpha \neq \beta$ ). We assume that the system is connected, namely that it is possible to reach any state from an arbitrary initial state in a finite number of transitions. We furthermore assume that when $R_{\beta \alpha}>0$ then also $R_{\alpha \beta}>0$ (microreversibility).

The probability $p_{\alpha}(t)$ to find the system in state $\alpha$ evolves according to a master equation

$$
\frac{d \boldsymbol{p}}{d t}=\boldsymbol{R} \boldsymbol{p},
$$

where the diagonal elements of $\boldsymbol{R}$ satisfy $R_{\alpha \alpha}=$ $-\sum_{\sigma \neq \alpha} R_{\sigma \alpha}$, thereby ensuring probability conservation.

It is often helpful to represent the jump process using a graph. The nodes of this graph correspond to the coarse-grained states, also referred to as sites. The links represent the possible (bidirectional) transitions between sites. An example of a graph representing a 4 site system with 5 possible transitions is depicted in Fig. 17.

The NPT was derived for thermally activated transitions with rates $R_{\beta \alpha}=k \exp \left[\left(E_{\alpha}-B_{\alpha \beta}\right) / T\right]$, where $E_{\alpha}$ are 
site energies and $B_{\alpha \beta}$ are barrier energies, while $T$ is the temperature of the environment. This parametrization of rates naturally describes certain molecular machines [3, 16], but can be used for any system satisfying detailed balance. When the set of parameters $\left\{E_{\alpha}, B_{\alpha \beta}\right\}$ do not vary with time the system relaxes to an equilibrium distribution with $p_{\alpha}^{(e q)} \propto e^{-E_{\alpha} / T}$. Note that detailed balance, $R_{\beta \alpha} p_{\alpha}^{(e q)}=R_{\alpha \beta} p_{\beta}^{(e q)}$, requires symmetric barriers, $B_{\alpha \beta}=B_{\beta \alpha}$.

When the system is driven by a time periodic variation of the site and barrier energies, with period $\tau$, Floquet theory states that the system settles into a time periodic asymptotic state [25]. In what follows we always assume that the system is in this asymptotic state.

During a cycle of the driving the probability can slosh back and forth between sites. As a result, the existence or lack thereof of net directed motion is determined by the integrated fluxes

$$
\phi_{\alpha \beta} \equiv \oint J_{\alpha \beta}(t) d t
$$

where $J_{\alpha \beta}(t)=R_{\alpha \beta}(t) p_{\beta}(t)-R_{\beta \alpha}(t) p_{\alpha}(t)$ is the momentary probability current flowing from $\beta$ to $\alpha$. The integral over time is taken over a full period of the driving cycle. A non vanishing $\phi_{\alpha \beta}$ for any pair of $\alpha, \beta$ means that the variation of parameters have resulted in some directed motion. For the catenanes of [16] the directed motion corresponds to rotation of the smaller ring like molecule along the larger molecule. More generally this directed motion will express the existence of some physical effect which can be utilised.

The NPT [18] states that one needs to vary both the barrier and site energies to generate directed motion. In particular, when one varies only $\left\{E_{\alpha}(t)\right\}$, while the $\left\{B_{\alpha \beta}\right\}$ are kept fixed, no directed motion is generated. This is non intuitive, since it seems natural that one should be able to induce directed motion by designing a driving cycle in which the most energetically favourable site changes in a cyclic way, for example between sites $\alpha \rightarrow \beta \rightarrow \delta \rightarrow \alpha$ in Fig. 1.

Many particle stochastic pumps- Consider a set of $N$ distinguishable particles which make stochastic jumps between a set of sites. The state of this many particle system is described by specifying the location of each particle, $\boldsymbol{X} \equiv\left(x_{1}, x_{2}, \cdots, x_{N}\right)$, where $x_{i}$ denotes the site in which the $i$ th particle resides. For example, in the 4 site system depicted in Fig. $1 \mathrm{H}, \boldsymbol{X} \equiv(\gamma, \delta)$ describes a state in which particle $1(2)$ is in site $\gamma(\delta)$ respectively.

Transitions between many particle states are made via jumps of a single particle. Instantaneous jumps of several particles are neglected. We therefore adopt a notation that matches the nature of transitions. Let $\boldsymbol{X}_{\mid k} \equiv$ $\left(x_{1}, x_{2}, \cdots, x_{k-1}, x_{k+1} \cdots x_{N}\right)$ denote the location of all particles except the $k$ th one. Then $\boldsymbol{X}_{\mid k} ; x_{k} \rightarrow x_{k}^{\prime}$ conveniently labels the transition out of $\boldsymbol{X}$ in which the $k$ th particle jumps to state $x_{k}^{\prime}$.
The fact that only single particle jumps connect the many particle states means that the graph representing the many particle stochastic pump is constructed by a Cartesian product of single particle graphs [26]. Fig. [1 (b) and (c) depicts the many particle (or product) graphs corresponding to two or three particles residing in the sites of Fig. 1h. It is clear that when $N \gg 1$ this product graph becomes quite complicated.

Which transition rates should one expects in this type of many particle system? For non interacting particles the total energy of the system is

$$
\mathcal{E}(\boldsymbol{X})=\sum_{\sigma} n_{\sigma}(\boldsymbol{X}) E_{\sigma},
$$

where the summation is over the sites, and $n_{\sigma}(\boldsymbol{X})$ is the number of particles residing in site $\sigma$. For any transition we can also assign a many particle energy barrier. The barrier for the transition $\boldsymbol{X}_{\mid k} ; \alpha \rightarrow \beta$ is

$$
\mathcal{B}\left(\boldsymbol{X}^{\prime}, \boldsymbol{X}\right)=\mathcal{E}\left(\boldsymbol{X}_{\mid k}\right)+B_{\beta \alpha} .
$$

The non-vanishing transition rates are then given by

$\mathcal{R}_{\boldsymbol{X}^{\prime}, \boldsymbol{X}}=\mathcal{R}_{\boldsymbol{X}_{\mid k} ; x_{k} \rightarrow x_{k}^{\prime}}=k \exp \left[\left(\mathcal{E}(\boldsymbol{X})-\mathcal{B}\left(\boldsymbol{X}^{\prime}, \boldsymbol{X}\right)\right) / T\right]$.

It is easy to verify that in absence of interactions this rate is given by $k \exp \left[\left(E_{\alpha}-B_{\beta \alpha}\right) / T\right]$, just like a single particle pump. The resulting dynamics of a pump with $N$ non-interacting particles is that of $N$ copies of a single particle pumps, see Sec. I of the supplementary material.

Interactions will modify the expressions for the many particle energies and barriers. We consider short range, local, interactions, so that a particle is only affected by particles residing at the same site. Within the same spirit, a particle which 'crosses the barrier' between sites does not interact with other particles. The transition rates are then given by (5) with

$$
\begin{aligned}
\mathcal{E}(\boldsymbol{X}) & =\sum_{\sigma} n_{\sigma}(\boldsymbol{X}) E_{\sigma}+U_{\sigma}\left(n_{\sigma}(\boldsymbol{X})\right), \\
\mathcal{B}\left(\boldsymbol{X}^{\prime}, \boldsymbol{X}\right) & =\sum_{\sigma} n_{\sigma}\left(\boldsymbol{X}_{\mid k}\right) E_{\sigma}+U_{\sigma}\left(n_{\sigma}\left(\boldsymbol{X}_{\mid k}\right)\right)+B_{\beta \alpha}(, 7)
\end{aligned}
$$

where $\mathcal{B}\left(\boldsymbol{X}^{\prime}, \boldsymbol{X}\right)=\mathcal{B}\left(\boldsymbol{X}, \boldsymbol{X}^{\prime}\right)$. We note that $\mathcal{E}(\boldsymbol{X})-$ $\mathcal{B}\left(\boldsymbol{X}^{\prime}, \boldsymbol{X}\right)=E_{\alpha}-B_{\beta \alpha}+\tilde{U}_{\alpha}\left(n_{\alpha}\right)$ with $\tilde{U}_{\alpha}\left(n_{\alpha}\right) \equiv U_{\alpha}\left(n_{\alpha}\right)-$ $U_{\alpha}\left(n_{\alpha}-1\right)$, recasting the rates in terms of single site quantities. When $\left\{E_{\alpha}\right\},\left\{U_{\alpha}\right\}$ do not vary in time the system relaxes to an equilibrium distribution with $p^{(e q)}(\boldsymbol{X}) \propto e^{-\mathcal{E}(\boldsymbol{X}) / T}$.

Since the energy of a single particle in a site is already accounted for, we must take $U_{\alpha}(0)=U_{\alpha}(1)=0$. Otherwise, we allow the interaction to take any value, implicitly assuming that it is not strong enough to destabilize or create sites.

Many particle fluxes and particle currents- We first examine the immediate application of the NPT to this many particle pump. The pump is driven by periodic 
variation of $\left\{E_{\alpha}(t)\right\}$ and $\left\{B_{\alpha \beta}(t)\right\}$. The NPT of [18] states that no directed motion is generated if either all many particle energies $\mathcal{E}(\boldsymbol{X})$, or alternatively all the $\mathcal{B}\left(\boldsymbol{X}^{\prime}, \boldsymbol{X}\right)$ are time independent. We emphasize that this result is not very useful, since i) the expression for $\mathcal{B}\left(\boldsymbol{X}^{\prime}, \boldsymbol{X}\right)$ mixes site energies and barriers, and ii) the integrated flux $\phi_{\boldsymbol{X}_{\mid k} ; x_{k} \rightarrow x_{k}^{\prime}}$ is not the observable one typically wishes to study, since it has the interpretation of the net probability probability flux that particle $k$ jumps from $x_{k}$ to $x_{k}^{\prime}$ conditioned on $\boldsymbol{X}_{\mid k}$.

It is much more natural to use the net current of particles flowing between two given sites as a measure of directed motion. This current is simply the sum of all many particle fluxes corresponding to a given transition

$$
\Phi_{\sigma \rightarrow \sigma^{\prime}}=\sum_{k=1}^{N} \sum_{\boldsymbol{X}_{\mid k}} \phi_{\boldsymbol{X}_{\mid k} ; \sigma \rightarrow \sigma^{\prime}}
$$

The main result of this letter is that the integrated particle currents (8) satisfy an additional NPT which is expressed in terms of the (single particle) site energies and barriers. It states that both $\left\{E_{\alpha}\right\}$ and $\left\{B_{\alpha \beta}\right\}$ need to be periodically varied in time in order to generate directed particle currents $\left(\Phi_{\sigma \rightarrow \sigma^{\prime}} \neq 0\right)$.

Derivation of NPT- When the $\left\{E_{\alpha}\right\}$ are time independent the system relaxes to an equilibrium state with no currents. We therefore focus on the case of time independent barriers and time dependent energies. One of the derivations of the single particle NPT, due to Mandal and Jarzynski 23], is based on incompatibility of two sets of equations, termed conservation laws and cycle equations. This graph based approach, which we employ here, will highlight the difference between the single particle graph, such as the one in Fig. 1a, and the many particle product graph. The derivation below is based on equations which hold on the much simpler one particle graph.

The first set of equations expresses conservation laws. For the many particle system the master equation can be rewritten as $\frac{d P(\boldsymbol{X})}{d t}=-\sum_{\boldsymbol{X}^{\prime} \neq \boldsymbol{X}} J_{\boldsymbol{X}^{\prime}, \boldsymbol{X}}$. The periodicity of $P(\boldsymbol{X}, t)$ can be used to obtain an equation $0=\sum_{\boldsymbol{X}^{\prime} \neq \boldsymbol{X}} \phi_{\boldsymbol{X}^{\prime}, \boldsymbol{X}}$ for any state $\boldsymbol{X}$. To derive equations which constrain the particle currents we examine the density of particles at the different sites

$$
\rho_{\sigma} \equiv \sum_{k=1}^{N} \sum_{\boldsymbol{X}_{\mid k}} P\left(x_{1}, x_{2}, \cdots, x_{k-1}, \sigma, x_{k+1} \cdots x_{N}\right) .
$$

We note that periodicity of $P$ implies periodicity of $\rho$. This leads to the conservation laws,

$$
\sum_{\sigma^{\prime} \neq \sigma} \Phi_{\sigma \rightarrow \sigma^{\prime}}=0
$$

expressing the fact that $\rho$ changes through particle currents. Eq. (10) is what one would naively guess, but it can also be derived from the conservation laws for many particle probabilities by explicitly performing the sum (91), see Sec. II of the supplementary information.

The second set of equations are cycle equations, defined on closed cycles of transitions on the graph representing the system. Let $\boldsymbol{X}^{\prime \prime} \rightarrow \boldsymbol{X} \rightarrow \boldsymbol{X}^{\prime}$ denote two consecutive transitions, such that in the first the $i^{\prime}$ th particle jumps from $x_{i}^{\prime \prime}$ to $x_{i}$, while in the second the $j$ 'th particle makes the $x_{j} \rightarrow x_{j}^{\prime}$ jump. We wish to examine the expression $e^{B_{x_{i}^{\prime \prime}, x_{i}} / T} J_{\boldsymbol{X}, \boldsymbol{X}^{\prime \prime}}+e^{B_{x_{j}^{\prime}, x_{j}} / T} J_{\boldsymbol{X}^{\prime}, \boldsymbol{X}}$, where we crucially use the single particle barriers. Our goal is to see when a sum of this form over a closed cycle will vanish. We focus on the two terms proportional to $P(\boldsymbol{X})$, given by, $-e^{B_{x_{i}^{\prime \prime}, x_{i}} / T} R_{\boldsymbol{X}^{\prime \prime}, \boldsymbol{X}} P(\boldsymbol{X})+$ $e^{B_{x_{j}^{\prime}, x_{j}} / T} R_{\boldsymbol{X}^{\prime}, \boldsymbol{X}} P(\boldsymbol{X})=\left[h\left(x_{j}\right)-h\left(x_{i}\right)\right] P(\boldsymbol{X})$, with $h(\alpha) \equiv \exp \left[\left(E_{\alpha}+\tilde{U}_{\alpha}\left(n_{\alpha}\right)\right) / T\right]$. This combination does not depend on the barriers. This is a result of the symmetry of the barriers, which follows from detailed balance 23]. It is clear that the terms cancel when $x_{i}=x_{j}$. Notably, they need not cancel when consecutive transition involve particle entering one site and then another particle leaving a different site. As a result not all the cycles on the product graph have cycle equations with single particle barriers as factors.

We now focus on a particular type of cycles, in which a given particle makes the transitions while the rest are essentially spectators. Lets assume that the $k^{\prime}$ th particle makes the transitions $\alpha_{1} \rightarrow \alpha_{2} \rightarrow \cdots \alpha_{L} \rightarrow \alpha_{1}$, closing a cycle on the corresponding single particle graph. In this case the sum discussed above has canceling terms, and

$$
\sum_{i=1}^{L} e^{B_{\alpha_{i+1}, \alpha_{i}} / T} J_{\boldsymbol{X}_{\mid k} ; \alpha_{i} \rightarrow \alpha_{i+1}}=0
$$

for any $k, \boldsymbol{X}_{\mid k}$. (Here $\alpha_{L+1}=\alpha_{1}$.) This equation holds even when the barriers vary with time.

For time independent barriers an integration of (11) over a period gives an equation for integrated fluxes. Moreover, since the same barriers appear for different $k$ 's, summation over $k, \boldsymbol{X}_{\mid k}$ gives

$$
\sum_{i=1}^{L} e^{B_{\alpha_{i+1}, \alpha_{i}} / T} \Phi_{\alpha_{i} \rightarrow \alpha_{i+1}}=0 .
$$

An equation of this type is valid for any closed cycle of transitions on the single particle graph.

We have shown that the integrated particle currents satisfy two sets of equations, the conservation laws (10), and the cycle equations (12). Both are defined using the nodes and links of the single particle graph (e.g. Fig. [1. regardless of the number of particles. The argument of 23], which was developed for single particle stochastic pumps, can be applied to Eqs. (10) and (12). The two sets of equations are incompatible and the only solution is $\Phi_{\alpha \rightarrow \beta}=0$. We conclude that the $\Phi_{\alpha \rightarrow \beta}$ satisfy an 


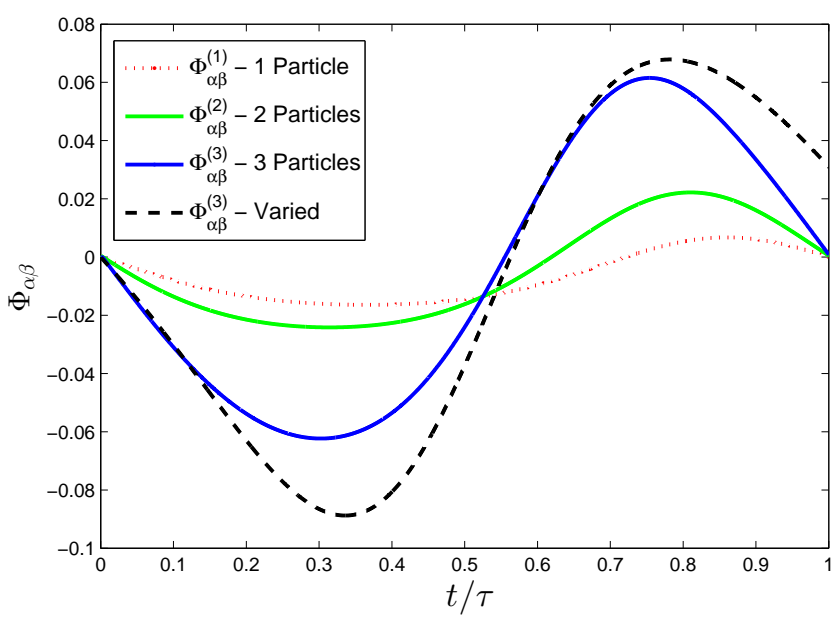

FIG. 2: Time integrated particle currents for 1,2 and 3 particles between sites $\beta \rightarrow \alpha$. Solid and dotted lines correspond to driving cycles with time independent barriers. The dashed line depicts the integrated current when one of the barriers is also varied in time.

NPT: One needs to vary both the single particle barriers, $\left\{B_{\beta \alpha}\right\}$, and site energies, $\left\{E_{\alpha}\right\}$, to generated non vanishing integrated particle currents. The interaction clearly plays a role similar to the site energies and therefore one could conceivably generate directed particle currents by varying the interaction and barriers in time.

Illustrative example- To illustrate our results we have investigated the system represented by Fig. 1 numerically. The system was driven using

$$
E_{i}(t)=-2+\cos \left(\frac{2 \pi}{\tau}\left(t+\frac{i-1}{4}\right)\right),
$$

where $i=1$ correspond to $\alpha, 2$ correspond to $\beta$, etc. The energy barriers were $B_{\alpha \beta}=-0.3, B_{\beta \gamma}=0, B_{\gamma \alpha}=$ $1, B_{\alpha \delta}=0.2$, and $B_{\gamma \delta}=-0.1$. When both energies and barriers were varied in time (dashed line in Fig. 2) $B_{\alpha \beta}(t)=\cos \left(\frac{2 \pi}{\tau}(t+0.11)\right)$, while the rest of the barriers were unchanged. The interaction were taken to be $U(2)=\ln 2$ and $U(3)=\ln 3$.

Fig. 2 compares the integrated particle currents, $\Phi_{\alpha \beta}(t) \equiv \int_{0}^{t} J_{\alpha \beta}\left(t^{\prime}\right) d t^{\prime}$, for a pump with 1,2 and 3 particles. For time independent barriers the integrated particle currents vanishes after a full period, as predicted by the NPT. (For one particle this is the NPT of [18].) The dashed curve demonstrates that directed motion is generated when both barriers and site energies are varied.

Fig. 3 depicts the integrated many particle probability fluxes corresponding to the $\beta \rightarrow \delta$ transition (fixed barriers). It is clear the $\phi$ 's need not vanish after full period, even when the NPT holds for the net particle currents $\Phi_{\sigma \rightarrow \sigma^{\prime}}$ (such as the dashed line in Fig. 3). This expresses the fact that not all cycles on the product graph have cycle equations.

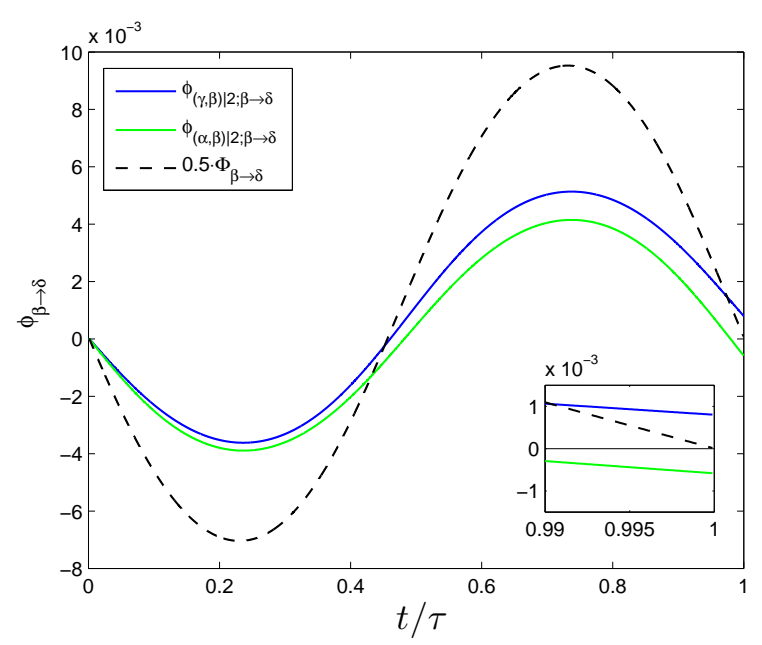

FIG. 3: Integrated fluxes corresponding to the $\beta \rightarrow \delta$ transitions in the two particle pump of $1(\mathrm{~b})$. Solid lines correspond to many particles fluxes. The dashed line depicts the integrated particle flux $\Phi_{\beta \rightarrow \delta}$ divided by 2 . The inset shows the region near $t=\tau$.

Summary- We have studied stochastic pumps with several interacting particles and found that the net particle current satisfies an NPT. We emphasize the NPT of [18] would give a condition on variation of many particle parameters $\{\mathcal{E}, \mathcal{B}\}$ while here the condition uses the more natural site parameters $\{E, B\}$.

Interactions in stochastic pumps are rarely considered. In 27] an open system with interacting particles was studied, and the large deviation function was shown to be equivalent to that of a non interacting system when branching ratios were time independent. The two papers deal with different setups. Our stochastic pumps conserve the number of particles and the NPT is not asymptotic in time. Nevertheless, in both systems a simplification was achieved for time-independent branching ratios. (Here for particles leaving the same site.) This hints that there is an underlying structure in such interacting systems which merits further study.

Weakly coupled triple quantum dots [28] offer a possible experimental realization of interacting particles obeying stochastic dynamics, especially when the temperature is larger than the mean level spacing. The system can be controlled by gate voltages, although the transition rates may differ from those considered here. A system of several colloidal particles in water offer another natural realization. Holographic techniques allow to create a controllable potential landscape [29, 30] for the colloids, including one composed of several adjacent traps. Moreover, particles in the same trap interact sterically.

Acknowledgements We are grateful for support from the US-Israel Binational Science Foundation (grant 2010363), and the Israel Science Foundation (grant 924/11). 
[1] J. Howard, Mechanics of Motor Proteins and the Cytoskeleton (Sinauer Associates, Sunderland, Massachusetts, 2001).

[2] G. S. Kottas, L. I. Clarke, D. Horinek, and J. Michl, Chemical Reviews 105, 1281 (2005).

[3] E. Kay, D. Leigh, and F. Zerbetto, Angewandte Chemie International Edition 46, 72 (2007).

[4] B. L. Feringa, The Journal of Organic Chemistry 72, 6635 (2007).

[5] J. Michl and E. C. H. Sykes, ACS Nano 3, 1042 (2009).

[6] A. Coskun, M. Banaszak, R. D. Astumian, J. F. Stoddart, and B. A. Grzybowski, Chem. Soc. Rev. 41, 19 (2012).

[7] P. Reimann, Physics Reports 361, 57 (2002).

[8] J. M. R. Parrondo, Phys. Rev. E 57, 7297 (1998).

[9] I. M. Sokolov, Journal of Physics A: Mathematical and General 32, 2541 (1999).

[10] R. D. Astumian, Phys. Rev. Lett. 91, 118102 (2003).

[11] N. A. Sinitsyn and I. Nemenman, Phys. Rev. Lett. 99, 220408 (2007).

[12] S. Rahav, Journal of Statistical Mechanics: Theory and Experiment 2011, P09020 (2011).

[13] V. Y. Chernyak, J. R. Klein, and N. A. Sinitsyn, The Journal of Chemical Physics 136, 154107 (2012).

[14] N. A. Sinitsyn, Journal of Physics A: Mathematical and Theoretical 42, 193001 (2009).

[15] R. D. Astumian, Annual Review of Biophysics 40, 289 (2011).

[16] D. A. Leigh, J. K. Y. Wong, F. Dehez, and F. Zerbetto,
Nature 424, 174 (2003).

[17] R. D. Astumian, Proceedings of the National Academy of Sciences 104, 19715 (2007).

[18] S. Rahav, J. Horowitz, and C. Jarzynski, Phys. Rev. Lett. 101, 140602 (2008).

[19] V. Y. Chernyak and N. A. Sinitsyn, Phys. Rev. Lett. 101, 160601 (2008).

[20] J. Horowitz and C. Jarzynski, Journal of Statistical Physics 136, 917 (2009).

[21] D. Mandal and C. Jarzynski, The Journal of Chemical Physics 137, 234104 (pages 7) (2012).

[22] C. Maes, K. NetoCny, and S. R. Thomas, The Journal of Chemical Physics 132, 234116 (pages 6) (2010).

[23] D. Mandal and C. Jarzynski, Journal of Statistical Mechanics: Theory and Experiment 2011, P10006 (2011).

[24] J. Ren, V. Y. Chernyak, and N. A. Sinitsyn, Journal of Statistical Mechanics: Theory and Experiment 2011, P05011 (2011).

[25] P. Talkner, New Journal of Physics 1, 4 (1999).

[26] W. Imrich, S. Klavzar, and D. F. Rall, Topics in Graph Theory: Graphs and Their Cartesian Product (Taylor \& Francis Inc, Natick, USA, 2008).

[27] V. Y. Chernyak, M. Chertkov, and N. A. Sinitsyn, Journal of Statistical Mechanics: Theory and Experiment 2011, P09006 (2011).

[28] C.-Y. Hsieh, Y.-P. Shim, M. Korkusinski, and P. Hawrylak, Rep. Prog. Phys, 75, 114501 (2012).

[29] M. Polin, K. Ladavec, S.-H. Lee, Y. Roichman, and D. G. Grier, Opt. Expr., 4, 5831 (2005).

[30] M. Yevnin, D. Kasimov, Y. Gluckman, Y. Ebenstein, and Y. Roichman, Biomed. Opt. Expr., 4, 2087 (2013). 